\title{
NGAL as an Early Predictive Marker of Diabetic Nephropathy in Children and Young Adults with Type 1 Diabetes Mellitus
}

\author{
Nektaria Papadopoulou-Marketou, ${ }^{1,2}$ Alexandra Margeli, ${ }^{3}$ Ioannis Papassotiriou, ${ }^{3}$ \\ George P. Chrousos, ${ }^{2}$ Christina Kanaka-Gantenbein, ${ }^{2}$ and Jeanette Wahlberg ${ }^{1}$ \\ ${ }^{1}$ Department of Endocrinology, Department of Medical and Health Sciences, Linkoping University, Linkoping, Sweden \\ ${ }^{2}$ Diabetes Centre, Department of Endocrinology, Diabetes and Metabolism, First Department of Pediatrics, National and \\ Kapodistrian University of Athens, Aghia Sophia Children's Hospital, Athens, Greece \\ ${ }^{3}$ Department of Clinical Biochemistry, Aghia Sophia Children's Hospital, Athens, Greece
}

Correspondence should be addressed to Nektaria Papadopoulou-Marketou; papadopoulounektaria@gmail.com

Received 1 September 2016; Accepted 3 April 2017; Published 23 May 2017

Academic Editor: Feng Wang

Copyright (c) 2017 Nektaria Papadopoulou-Marketou et al. This is an open access article distributed under the Creative Commons Attribution License, which permits unrestricted use, distribution, and reproduction in any medium, provided the original work is properly cited.

\begin{abstract}
Aims. Type 1 diabetes (T1D) is often associated with early microvascular complications. Previous studies demonstrated that increased systolic (SAP) and diastolic arterial blood pressures (DAP) are linked to microvascular morbidity in T1D. The aim of the study was to investigate the predictive role of neutrophil gelatinase-associated lipocalin (NGAL) in unravelling early cardiorenal dysfunction in T1D. Methods. Two T1D patient groups participating in two-centre prospective cohorts were studied. Group A consisted of 57 participants aged 13.9 years (SD: 3.1) and group B consisted of 59 patients aged 28.0 years (SD: 4.4). Forty-nine healthy children [age: 10.5 years (SD: 6.6)] and 18 healthy adults [age 27.7 years (SD: 4.2)] served as controls. Serum concentrations of NGAL (ELISA) were determined, and SAP and DAP were examined (SAP and DAP also expressed as $z$-scores in the younger group). Results. NGAL correlated positively with SAP in both patient groups $(P=0.020$ and $P=0.031$, resp.) and SAP $z$-score $(P=0.009)$ (group A) and negatively with eGFR in both groups $(P<0.001$ and $P<0.001$, resp.). Conclusions. NGAL may be proposed as a biomarker of early renal dysfunction even in nonalbuminuric T1D patients, since it was strongly associated with renal function decline and increasing systolic arterial pressure even at prehypertensive range in people with $\mathrm{T} 1 \mathrm{D}$, in a broad age range.
\end{abstract}

\section{Introduction}

Type 1 diabetes mellitus (T1D) is a prevalent autoimmune disease in childhood and young adulthood. Diabetes nephropathy (DN) is a chronic devastating complication associated with an increased risk of end-stage renal failure, as well as cardiovascular disease and premature death. It has been previously reported that childhood-onset T1D is associated with a 4 -fold increase in the overall standardized mortality rate [1].

In both the USA and Europe, approximately 20\% of T1D persons develop DN and progress to end-stage renal disease (ESRD). Since T1D often occurs in younger ages, ESRD most often develops at an earlier age, during the most productive years of persons with the disease. Thus, it represents a significant burden to the patients and the society they live in $[2,3]$. Moreover, nonalbuminuric $\mathrm{DN}$ has been reported to have a prevalence of $2 \%$ among people with T1D and chronic kidney disease (CKD) $[4,5]$.

Nowadays, the screening of $\mathrm{DN}$ is based on microalbuminuria (MA) assessment [6], and MA may be found in $12-16 \%$ of adolescents with T1D [7]. In early stages, regression to nonalbuminuria is frequently observed [8]. Puberty itself, as well as poor glycaemic control, is an independent risk factor for MA in persons with T1D [9]. However, the diagnostic value of microalbuminuria in DN has recently been challenged by a large number of researchers worldwide, while it has been widely proposed that other biomarkers are needed for the early identification of diabetic renal lesions $[2,5,10,11]$. Previous studies have provided evidence of several clinical 
and laboratory predictors for $\mathrm{DN}$, such as increased systolic arterial blood pressure (SAP), even within the prehypertensive range [12], and dyslipidaemia, identifying people at risk for early endotheliopathy and cardiovascular disease (CVD) as well [13]. Among T1D individuals with nephropathy and hypertension, $50 \%$ will progress to end-stage renal disease within a decade [6].

The pathophysiologic changes in DN linked to renal function decline are associated with cellular and extracellular derangements in both the glomerular and tubular compartments [14]. Several studies have reported that nonalbuminuric subjects, including prepubertal children with long-standing diabetes, often have glomerular basement membrane (GBM) thickening, mesangial expansion $[15,16]$, and significant glomerulopathy lesions $[17,18]$. Glomerular and renal tubular interstitial injury plays a role in the pathogenesis of $\mathrm{DN}[19]$, and various tubular markers have been assessed in the early detection of $\mathrm{DN}[10,20]$. Among them, neutrophil gelatinase-associated lipocalin (NGAL), first purified and identified in 1993 by Kjeldsen et al., seems to be a promising biomarker $[19,21]$. NGAL is a 178 -amino acid $25 \mathrm{kDa}$ protein that belongs to the lipocalin protein family. It is primarily produced in renal tubules in response to structural kidney injury [22].

In contrast to conventional markers, such as serum creatinine, blood urea nitrogen, or serum cystatin C (CysC), NGAL is not considered a marker of renal function, but rather reflects structural damage of renal cells. In previous studies, NGAL was reported as effective in the early diagnosis of acute kidney injury (AKI) in several clinical settings [2325 ] and was also validated as a significant prognostic factor in cardiovascular morbidity [26]. The association between the early tubular lesions in nonalbuminuric patients with T1D and NGAL was further supported by recently published studies $[18,19,21]$.

The aim of this study was to determine the possible predictive role of serum NGAL as a supplementary marker to urinary albumin excretion, in unmasking early renal structural injury, renal function decline, and cardiovascular risk in asymptomatic, normotensive individuals in two different age groups, childhood and adulthood, with various duration of T1D and irrespective of the presence of microalbuminuria.

\section{Materials and Methods}

Two observational cross-sectional prospective long-term follow-up studies took place in two university diabetes centres.

The group A consisted of 57 participants with T1D with a mean age of 13.9 years (SD: 3.1 ) and a mean diabetes duration of 5.4 years (SD: 3.3) at the time of the evaluation, who were prospectively followed at the Diabetes Centre of the First Department of Pediatrics of the University of Athens, Greece (Table 1) [18].

The group B consisted of 59 young adults with T1D with a mean age of 28.0 years (SD: 4.4) and a mean diabetes duration of 7.4 years (SD: 1.9) at the time of the evaluation, who were prospectively followed for at least 5 years at the Department of Endocrinology of the University Hospital of Linkoping,
Sweden (Table 1) [27]. The diagnosis of T1D was based in all participating patients on the presence of the positive titer of at least one of the known circulating, islet-specific, pancreatic autoantibodies related to T1D (autoantibodies against glutamic acid decarboxylase); the $40 \mathrm{~K}$ fragment of tyrosine phosphatase (IA2); or insulin antibodies. Regarding group A, eight of the patients presented with microalbuminuria at inclusion in the study, while five of them had known persistent microalbuminuria. In group B, seven out of 59 patients had persistent microalbuminuria at enrolment.

Forty-nine healthy children with a mean age of 10.57 (SD: 6.6) served as controls (group C) (Table 1). Informed consent was obtained from all the participants and their parents before their inclusion in the study. Eighteen healthy adults with a mean age of 27.7 (SD: 4.2) also served as controls (group D).

Both studies were approved by the respective Ethics Committee (Ethics Committee of the Aghia Sophia Children's Hospital in Athens, Greece, and Ethics Committee of the Medical Faculty in Linkoping, Sweden, resp.).

Inclusion criteria for all participants were T1D while exclusion criteria were the presence of active urinary tract infection, glucocorticoid medication, antihypertensive treatment, pregnancy, renal disease, and any chronic disease besides T1D.

Estimated glomerular filtration rate (eGFR) was calculated using the most recently suggested formula by Grubb et al. $\left(\mathrm{eGFR}=130 \times\right.$ cystatin $\mathrm{C}^{-1.069} \times$ age $\left.^{-0.117}-7\right)[28]$.

Serum and urinary NGAL levels were measured using a commercially available ELISA (Bioporto, Gentofte, Denmark). The reference range in plasma was $37-106 \mathrm{ng} / \mathrm{ml}$, and the intra- and interassay coefficients of variation were $5.6 \%$ and $6.4 \%$, respectively.

Cystatin $\mathrm{C}$ concentration was determined by an immunonephelometric technique using the BN Prospec nephelometer (Dade Behring, Siemens Healthcare Diagnostics, Liederbach, Germany). The interassay coefficient of variation for the assay was $5.05 \%$ and $4.87 \%$ at mean concentrations of 0.97 and $1.90 \mathrm{mg} / \mathrm{l}$, respectively, and the reference range in plasma was $0.47-1.09 \mathrm{mg} / \mathrm{l}$.

Statistical analyses were performed using MedCalc, version 12.5 (MedCalc Software, Ostend, Belgium). Correlation analysis was used to determine whether the values of the two variables are associated using Pearson parametric correlation. Student $t$-test was performed (paired samples $t$-test was used in order to test the null hypothesis that the average of the deviations between a series of paired observations is zero when carried out on the same individuals or independent samples $t$-test when performed between the patient and control groups). Multiple regression analysis was used to test the relation between one dependent variable and at least one independent variables. The significance was defined as a $P$ value $<0.05$, rho, and $95 \%$ confidence interval (CI) for the correlation coefficient.

\section{Results}

The mean value for NGAL in group A was $67.6 \mathrm{ng} / \mathrm{ml}$ (SD: 27.9) while in group B, it was $85.2 \mathrm{ng} / \mathrm{ml}$ (SD: 29.4) 


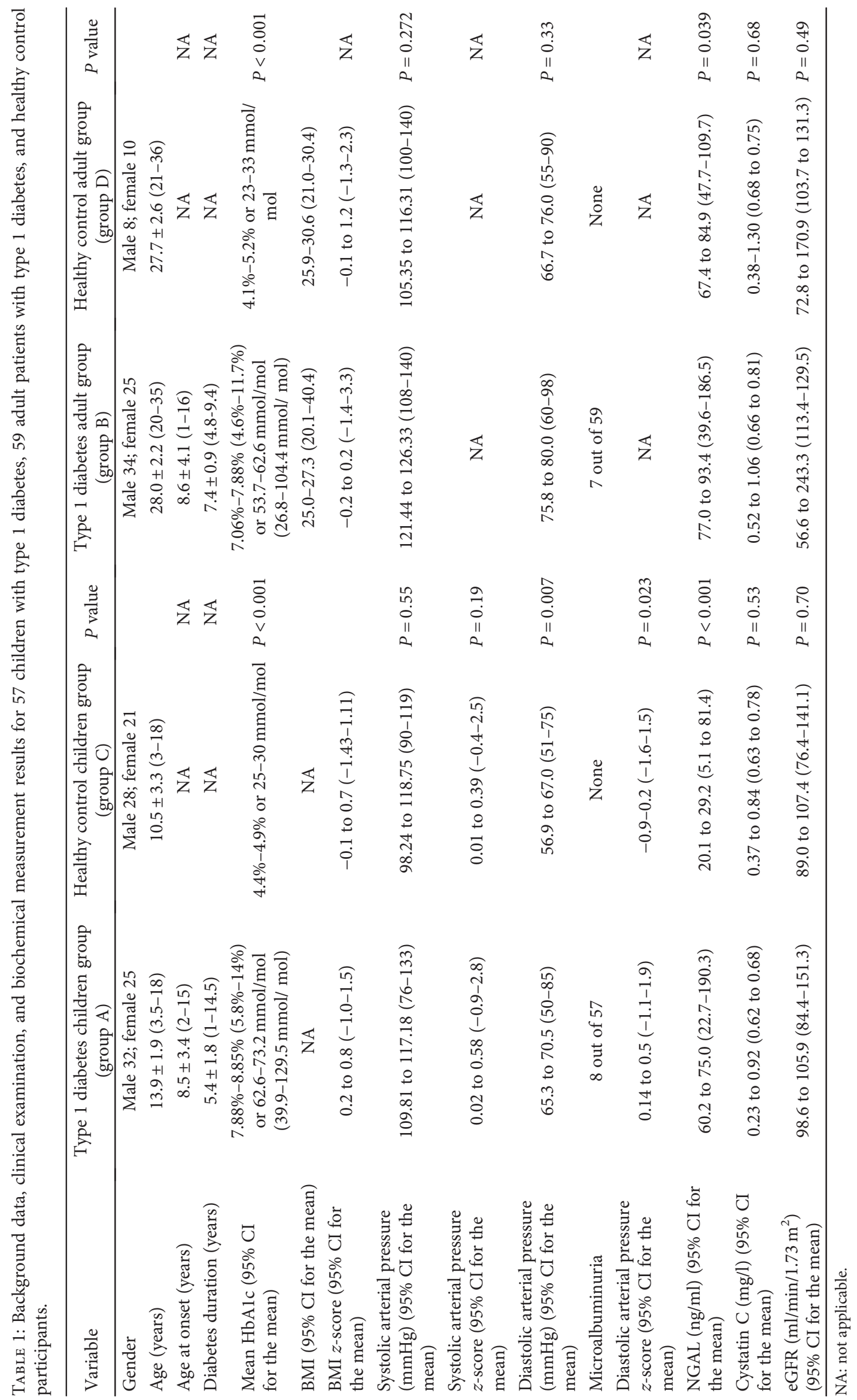




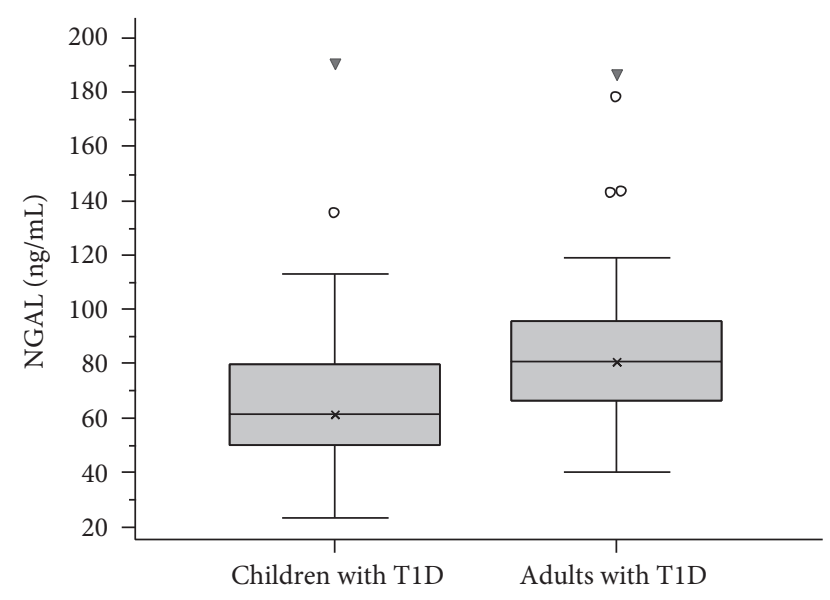

Figure 1: Box plot of NGAL levels in children and adults with T1D. Boxes represent the interquartile range; lines inside the boxes represent the median value; cross represents mean marker; and whiskers represent the lowest and highest observations, respectively. Children group had statistically significant lower median value than the adult group $(P<0.001)$.

$(P<0.001)$ (Figure 1). Both groups had a significantly higher mean value compared with the two control groups [mean NGAL: $24.6 \mathrm{ng} / \mathrm{ml}$ (SD: 15.8) for group $\mathrm{C}$ and $76.1 \mathrm{ng} / \mathrm{ml}$ (SD: 19.2) for group D], according to paired $t$-test analysis for independent groups $(P<0.001$ and $P=0.039$, resp.) (Figure 2).

The mean value for cystatin $\mathrm{C}$ in group A was $0.65 \mathrm{ng} / \mathrm{ml}$ (SD: 0.1 ) while in group B, it was $0.71 \mathrm{mg} / \mathrm{l}$ (SD: 0.1 ). Both groups had no significantly higher mean value compared with the two control groups [mean cystatin C: $0.68 \mathrm{ng} / \mathrm{ml}$ (SD: 0.1 ) for group $\mathrm{C}$ and $0.74 \mathrm{ng} / \mathrm{ml}$ (SD: 0.02 ) for group $\mathrm{D}]$ per paired $t$-test analysis for independent groups.

Regression analysis revealed that NGAL had a negative correlation with eGFR in both group A $(r=-0.501$, $P<0.001)$ and group $\mathrm{B}(r=-0,418, P<0.001)$. Regression analysis of NGAL levels and eGFR values, adjusted for age in both groups $\mathrm{A}$ and $\mathrm{B}$ with type 1 diabetes, showed a significant correlation between the biomarker and renal function decline $(F$ ratio $=5.93, P=0.0037)$ (Figure 3$)$. No significant correlation was found between NGAL and eGFR in either control groups.

NGAL was positively correlated with systolic arterial pressure, according to logarithmic curve regression equations in both patient groups $(P=0.020$ for group $\mathrm{A}$ and $P=0.031$ for group $\mathrm{B})$. In children group $\mathrm{A}$, SAP $z$-score was also analysed and a positive correlation with NGAL was revealed $(P=0.009)$. Regression analysis adjusted for age showed a significant positive correlation of NGAL with systolic arterial pressure in both groups with type 1 diabetes ( $F$ ratio $=17.1, P=0.0001$ ) (Figure 4$)$. No significant correlation between NGAL and either DAP or DAP $z$-score was noted. NGAL was not significantly correlated with SAP, SAP $z$-score, DAP, or DAP $z$-score in the control groups.

Urinary NGAL was analysed, but no significant correlation with neither SAP nor eGFR was revealed.

Neither microalbuminuria nor HbA1c had any significant correlations with NGAL in either group.

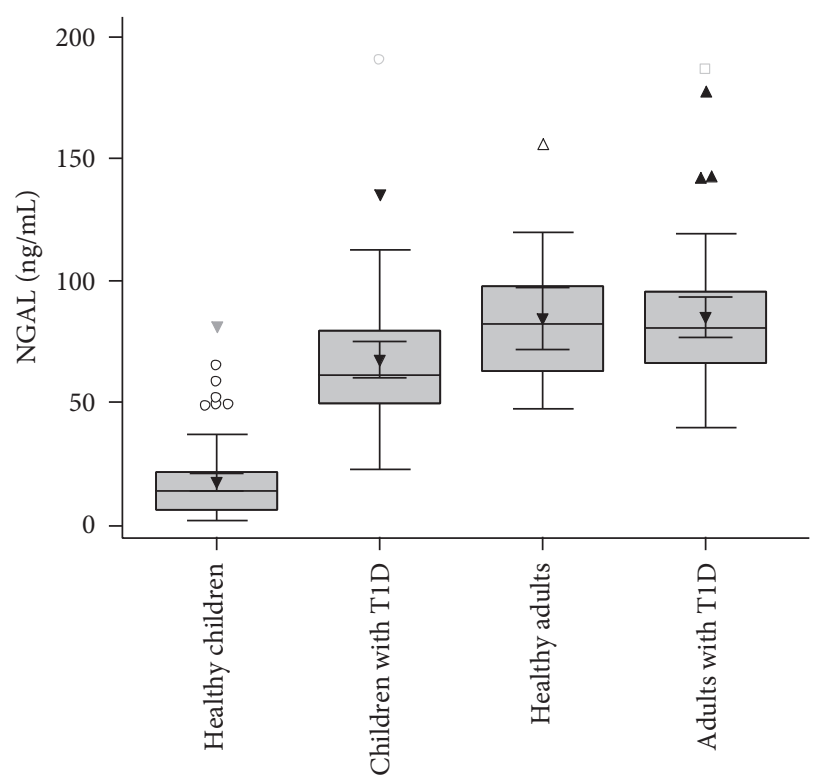

FIgURE 2: Box plot of NGAL levels. Boxes represent the interquartile range; lines inside the boxes represent the median value; cross represents mean marker; and whiskers represent the lowest and highest observations, respectively. The mean value for NGAL in children with T1D was $67.67 \mathrm{ng} / \mathrm{ml}$ (SD: 27.93) while in the group of adults with T1D, it was $85.26 \mathrm{ng} / \mathrm{ml}$ (SD: 29.49). Both groups had a significantly higher mean value compared with the two control groups (mean NGAL: $24.69 \mathrm{ng} / \mathrm{ml}$ (SD: 15.89) for the children control group $(P<0.001)$ and $76.18 \mathrm{ng} / \mathrm{ml}(\mathrm{SD}: 19.22)$ for the adult control group $(P=0.039))$ according to paired $t$-test analysis for independent samples.

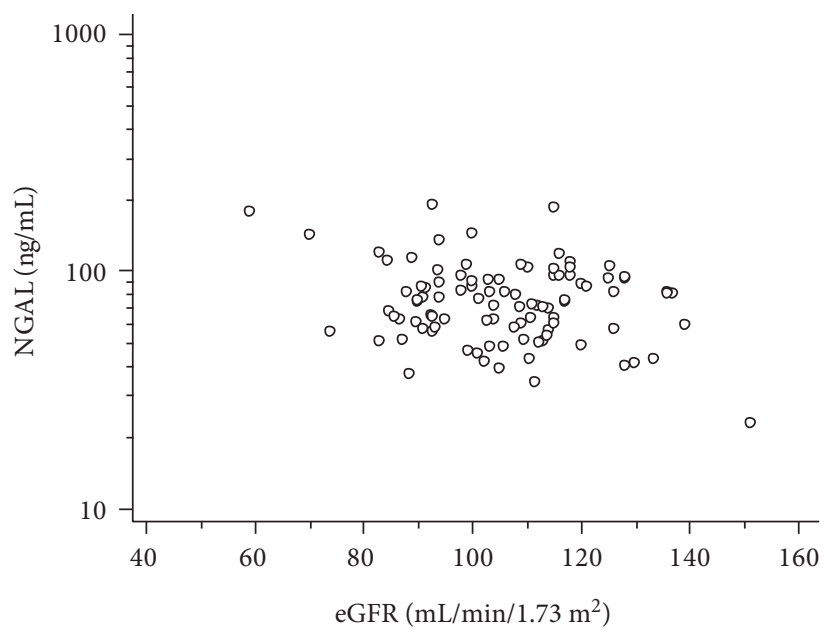

FIGURE 3: Serum NGAL had a negative correlation with eGFR values according to regression analysis, adjusted for age in both children and adult groups with type 1 diabetes $(F$ ratio $=5.93$, $P=0.0037)$.

\section{Discussion}

Microalbuminuria has been considered the earliest marker of the development of diabetes nephropathy (DN) and is often linked to established significant glomerular damage, 


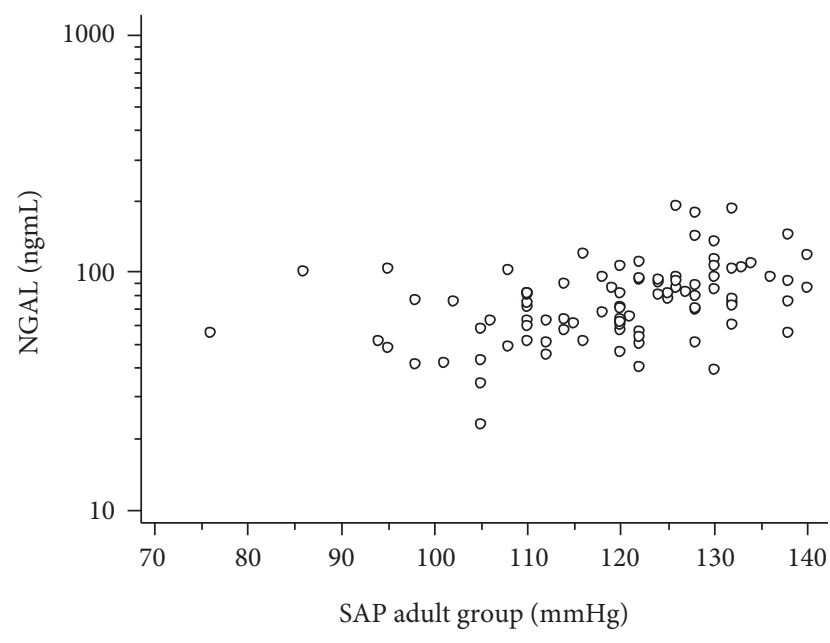

FIgURE 4: Serum NGAL had a significant positive correlation with systolic arterial pressure for children and adult groups with type 1 diabetes according to regression analysis adjusted for age $(F$ ratio $=17.1, P=0.0001)$.

traditionally believed to be the most common lesion in type $1 \mathrm{DN}$. However, recent studies showed that MA might be transient and not necessarily reflect permanent renal impairment [8]. Further recent reports suggested that pathophysiologic changes in DN include renal function decline associated with both cellular and extracellular derangements in glomerulotubular compartments [17, 18]. Besides, several lines of evidence suggest that early lesions in both glomerular and tubular structures may be present in nonalbuminuric patients. In line with this, cohort studies, including prepubertal children with average diabetes duration of 5-8 years, revealed glomerular base membrane thickening and mesangial expansion [16], while they also disclosed that longstanding nonalbuminuric T1D participants might have significant glomerulopathy lesions $[17-19,21]$. Moreover, the prevalence of nonalbuminuric CKD in T1D was recently reported to be $2 \%$ and was associated with a higher risk of cardiovascular morbidity as well as all-cause mortality in people with T1D $[4,5]$.

The increased hyperglycaemia-induced permeability of the glomeruli that is associated with hyperfiltration and microalbuminuria is not debated, but the perception of microalbuminuria being the only marker for diagnosing and excluding the development of diabetic nephropathy needs to be further investigated, to reduce the number of the young T1D normoalbuminuric people who will progress to CKD. According to the National Kidney Foundation (NKF), a patient is considered to be diagnosed with chronic kidney disease (CKD) if he or she presents a GFR $<60 \mathrm{ml} / \mathrm{min}$ for three months or more. Alternatively, other ongoing structural or functional renal abnormalities, which can be detected by pathological abnormalities or specific markers, are considered CKD [29]. Besides, early renal function decline, defined as a progressive loss of GFR over time even if it remains within the normal range, is also supported to be associated with DN in T1D [30].

Our study revealed strong negative correlations between serum NGAL and eGFR in both adult and children groups, indicating a significant association with renal function decline. No significant associations between urinary NGAL and eGFR were found in this study, and this finding does not agree with previous reports.

This finding underpins the value of NGAL as a biomarker of early renal damage in T1D, since NGAL had a positive correlation with the duration of T1D, mainly when eGFR was decreased, and this finding may reflect a progress of the early renal structural damage occurring during the disease, even if eGFR remains within the normal range. This finding is probably suggestive that NGAL is associated with the occurrence of ERFD. The number of the participants with T1D who presented microalbuminuria was much lower than the number of patients who presented a decreased eGFR. Moreover, the fact that a percentage of nonalbuminuric patients, who were found to have renal function decline, had higher NGAL levels, while some patients with microalbuminuria had normal NGAL values, may suggest that these two biomarkers, that is, microalbuminuria and NGAL, may reflect other sites of renal injury during the process of DN establishment. The link between the early tubular damage in nonalbuminuric people with T1D and increased NGAL is further supported by recently published studies $[15,17]$. The mean value of NGAL was significantly higher in the adult patient group. Besides, regression analysis revealed a correlation between age and the duration of type 1 diabetes in both children and adult. Urinary NGAL was also analysed in the paediatric participants, but no significant correlation with either GFR or SAP was found, and this finding does not agree with previous reports [31].

Systolic arterial pressure has been previously suggested as a predictor of DN [3]. In our study, since it was particularly focused on a young population, we estimated both SAP $z$-score and SAP for children and SAP for adults. We found that in two different age groups with T1D of various disease durations, NGAL correlated positively with increasing systolic arterial pressure, even if the latter remained within the prehypertensive or normal range. It has been previously reported that ambulatory blood pressure modestly rising in T1D patients is associated with the silent phase of DN up to 5 years before microalbuminuria appears [13]. Also, past studies had shown an association between renohypertensive diseases and NGAL [31]. Our findings may suggest that the association between NGAL and increasing SAP could reflect the early asymptomatic stages of DN progression in T1D and unravel possible underlying early renal injury. Since we found no similar significant associations in the control groups, our hypothesis is that the findings revealed from the two patient groups reflect the underlying microvascular pathogenesis in type 1 diabetes. Diabetic nephropathy has been linked with biomarker changes consistent with generalized endothelial dysfunction. Markers of endothelial dysfunction and arterial stiffness have also been correlated with MA in diabetic populations [16]. The association between NGAL and increasing SAP may suggest an indirect predictive role of NGAL as a cardiovascular morbidity marker. Undoubtedly, further studies investigating the endothelial dysfunction will further delineate the extent of microvascular damage in DN. 


\section{Conclusions}

To our knowledge, this is the first study that aimed at assessing the predictive value of other early markers of renal injury, besides microalbuminuria, such as NGAL in two different age groups of patients with T1D and found similar results. Moreover, a predictive role of NGAL has been demonstrated, as an early marker of diabetes nephropathy and probably asymptomatic cardiovascular morbidity independent of microalbuminuria.

Defining new predictive markers as supplementary tests to urinary albumin excretion for the early diagnosis of microvascular complications in T1D would lead to effective management and treatment approaches in time, which are needed to minimize the rates of severe reno- and cardiovascular as well as all-cause-associated morbidity in young people with T1D. Therefore, these data need to be further confirmed by large-scale longitudinal studies before being integrated into the risk assessment of follow-up of people with T1D.

\section{Conflicts of Interest}

The authors declare that they have no conflicts of interest.

\section{Acknowledgments}

The authors are grateful to Ioanna Kosteria and Chrysanthi Skevaki for their valuable contribution.

\section{References}

[1] G. A. Nichols, S. Vupputuri, and H. Lau, "Medical care costs associated with progression of diabetic nephropathy," Diabetes Care, vol. 34, no. 11, pp. 2374-2378, 2011.

[2] B. A. Perkins and A. S. Krolewski, "Early nephropathy in type 1 diabetes: a new perspective on who will and who will not progress," Current Diabetes Reports, vol. 5, no. 6, pp. 455-463, 2005.

[3] K. Raile, A. Galler, S. Hofer et al., "Diabetic nephropathy in 27,805 children, adolescents, and adults with type 1 diabetes: effect of diabetes duration, A1C, hypertension, dyslipidemia, diabetes onset, and sex," Diabetes Care, vol. 30, no. 10, pp. 2523-2528, 2007.

[4] L. M. Thorn, D. Gordin, V. Harjutsalo et al., "The presence and consequence of nonalbuminuric chronic kidney disease in patients with type 1 diabetes," Diabetes Care, vol. 38 , no. 11, pp. 2128-2133, 2015.

[5] N. Papadopoulou-Marketou, G. P. Chrousos, and C. KanakaGantenbein, "Diabetic nephropathy in type 1 diabetes: a review of early natural history, pathogenesis, and diagnosis," Diabetes/Metabolism Research and Reviews, vol. 33, no. 2, article e2841, 2017.

[6] C. E. Mogensen, "How to protect the kidney in diabetic patients: with special reference to IDDM," Diabetes, vol. 46, Supplement 2, pp. S104-S111, 1997.

[7] C. J. Schultz, T. Konopelska-Bahu, R. N. Dalton et al., "Microalbuminuria prevalence varies with age, sex, and puberty in children with type 1 diabetes followed from diagnosis in a longitudinal study. Oxford Regional
Prospective Study Group," Diabetes Care, vol. 22, no. 3, pp. 495-502, 1999.

[8] B. A. Perkins, L. H. Ficociello, K. H. Silva, D. M. Finkelstein, J. H. Warram, and A. S. Krolewski, "Regression of microalbuminuria in type 1 diabetes," The New England Journal of Medicine, vol. 348, no. 23, pp. 2285-2293, 2003.

[9] O. Access, "Adolescent type 1 Diabetes Cardio-renal Intervention Trial (AdDIT)," BMC Pediatrics, vol. 9, p. 79, 2009.

[10] A. Lacquaniti, V. Donato, B. Pintaudi et al., "“Normoalbuminuric" diabetic nephropathy: tubular damage and NGAL," Acta Diabetologica, vol. 50, no. 6, pp. 935-942, 2013.

[11] P. Hovind, P. Rossing, L. Tarnow, R. J. Johnson, and H. H. Parving, "Serum uric acid as a predictor for development of diabetic nephropathy in type 1 diabetes: an inception cohort study," Diabetes, vol. 58, no. 7, pp. 1668-1671, 2009.

[12] T. J. Orchard, K. Y. Forrest, L. H. Kuller, and D. J. Becker, "Lipid and blood pressure treatment goals for type 1 diabetes: 10-year incidence data from the Pittsburgh Epidemiology of Diabetes Complications Study," Diabetes Care, vol. 24, no. 6, pp. 1053-1059, 2001.

[13] P. L. Poulsen, K. W. Hansen, and C. E. Mogensen, “Ambulatory blood pressure in the transition from normo- to microalbuminuria. A longitudinal study in IDDM patients," Diabetes, vol. 43, no. 10, pp. 1248-1253, 1994.

[14] R. E. Gilbert and M. E. Cooper, "The tubulointerstitium in progressive diabetic kidney disease: more than an aftermath of glomerular injury?" Kidney International, vol. 56, no. 5, pp. 1627-1637, 1999.

[15] J. M. Steinke, A. R. Sinaiko, M. S. Kramer, S. Suissa, B. M. Chavers, and M. Mauer, "The early natural history of nephropathy in type 1 diabetes: III. Predictors of 5-year urinary albumin excretion rate patterns in initially normoalbuminuric patients," Diabetes, vol. 54, no. 7, pp. 2164-2171, 2005.

[16] E. N. Ellis, B. A. Warady, E. G. Wood et al., "Renal structuralfunctional relationships in early diabetes mellitus," Pediatric Nephrology (Berlin, Germany), vol. 11, no. 5, pp. 584-591, 1997.

[17] T. B. Torbjörnsdotter, N. E. S. S. Perrin, G. A. Jaremko, and U. B. Berg, "Widening of foot processes in normoalbuminuric adolescents with type 1 diabetes," Pediatric Nephrology (Berlin, Germany), vol. 20, no. 6, pp. 750-758, 2005.

[18] N. Papadopoulou-Marketou, C. Skevaki, I. Kosteria et al., "NGAL and cystatin C: two possible early markers of diabetic nephropathy in young patients with type 1 diabetes mellitus: one year follow up," Hormones (Athens, Greece), vol. 14, no. 2, pp. 232-240, 2015.

[19] F. L. Nauta, W. E. Boertien, S. J. L. Bakker et al., "Glomerular and tubular damage markers are elevated in patients with diabetes," Diabetes Care, vol. 34, no. 4, pp. 975-981, 2011.

[20] H. W. Chae, J. I. Shin, A. R. Kwon, H. S. Kim, and D. H. Kim, "Spot urine albumin to creatinine ratio and serum cystatin $\mathrm{C}$ are effective for detection of diabetic nephropathy in childhood diabetic patients," Journal of Korean Medical Science, vol. 27, no. 7, pp. 784-787, 2012.

[21] K. M. Thrailkill, C. S. Moreau, G. E. Cockrell et al., "Disease and gender-specific dysregulation of NGAL and MMP-9 in type 1 diabetes mellitus," Endocrine, vol. 37, no. 2, pp. 336-343, 2010.

[22] K. M. Schmidt-Ott, K. Mori, J. Y. Li et al., "Dual action of neutrophil gelatinase-associated lipocalin," Journal of the American Society of Nephrology : JASN, vol. 18, no. 2, pp. 407-413, 2007. 
[23] M. Haase, R. Bellomo, P. Devarajan, P. Schlattmann, A. HaaseFielitz, and N. M. A. I. Grp, "Accuracy of neutrophil gelatinase-associated lipocalin (NGAL) in diagnosis and prognosis in acute kidney injury: a systematic review and meta-analysis," American Journal of Kidney Diseases, vol. 54, no. 6, pp. 1012-1024, 2009.

[24] A. Haase-Fielitz, M. Haase, and P. Devarajan, "Neutrophil gelatinase-associated lipocalin as a biomarker of acute kidney injury: a critical evaluation of current status," Annals of Clinical Biochemistry, vol. 51, Part 3, pp. 335-351, 2014.

[25] M. Haase, R. Bellomo, P. Devarajan et al., "Novel biomarkers early predict the severity of acute kidney injury after cardiac surgery in adults," The Annals of Thoracic Surgery, vol. 88, no. 1, pp. 124-130, 2009.

[26] R. Hawkins, "New biomarkers of acute kidney injury and the cardio-renal syndrome," The Korean Journal of Laboratory Medicine, vol. 31, no. 2, pp. 72-80, 2011.

[27] L. Hyllienmark, N. Alstrand, B. Jonsson, J. Ludvigsson, G. Cooray, and J. Wahlberg-Topp, "Early electrophysiological abnormalities and clinical neuropathy: a prospective study in patients with type 1 diabetes," Diabetes Care, vol. 36, no. 10, pp. 3187-3194, 2013.

[28] A. Grubb, M. Horio, L. O. Hansson et al., "Generation of a new cystatin C-based estimating equation for glomerular filtration rate by use of 7 assays standardized to the international calibrator," Clinical Chemistry, vol. 60, no. 7, pp. 974-986, 2014.

[29] K. Uhlig and K. U. Eckardt, "A decade after the KDOQI CKD guidelines: impact on CKD guidelines," American Journal of Kidney Diseases : The Official Journal of the National Kidney Foundation, vol. 60, no. 5, pp. 705-706, 2012.

[30] A. S. Krolewski, M. A. Niewczas, J. Skupien et al., "Early progressive renal decline precedes the onset of microalbuminuria and its progression to macroalbuminuria," Diabetes Care, vol. 37, no. 1, pp. 226-234, 2014.

[31] A. Eirin, M. L. Gloviczki, H. Tang et al., "Chronic renovascular hypertension is associated with elevated levels of neutrophil gelatinase-associated lipocalin," Nephrology, Dialysis, Transplantation, vol. 27, no. 11, pp. 4153-4161, 2012. 


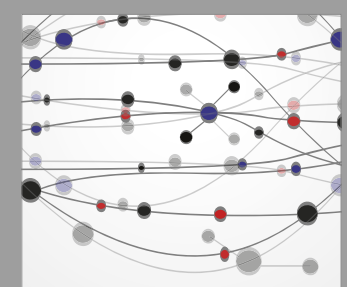

The Scientific World Journal
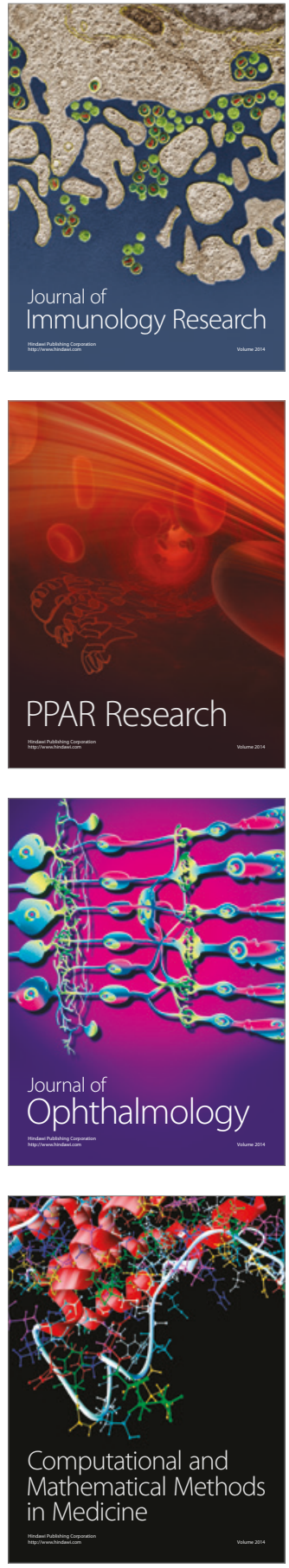

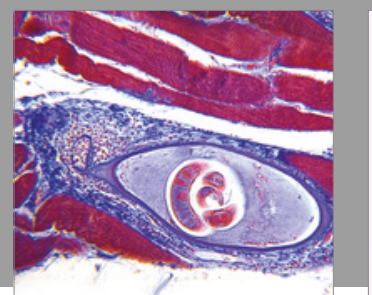

Gastroenterology Research and Practice
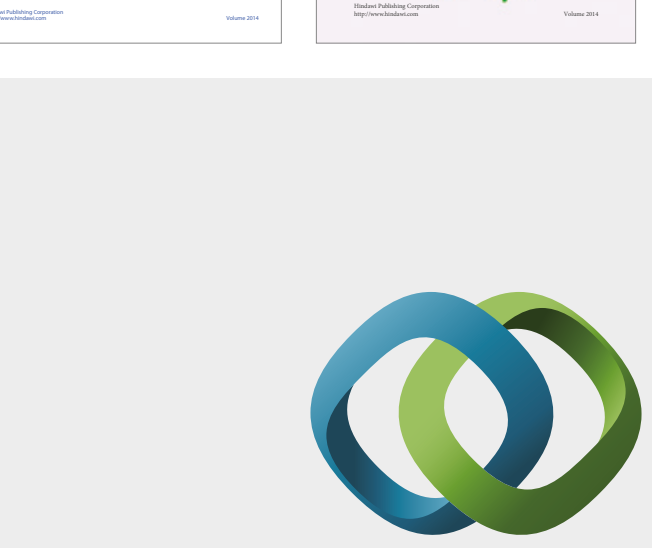

\section{Hindawi}

Submit your manuscripts at

https://www.hindawi.com
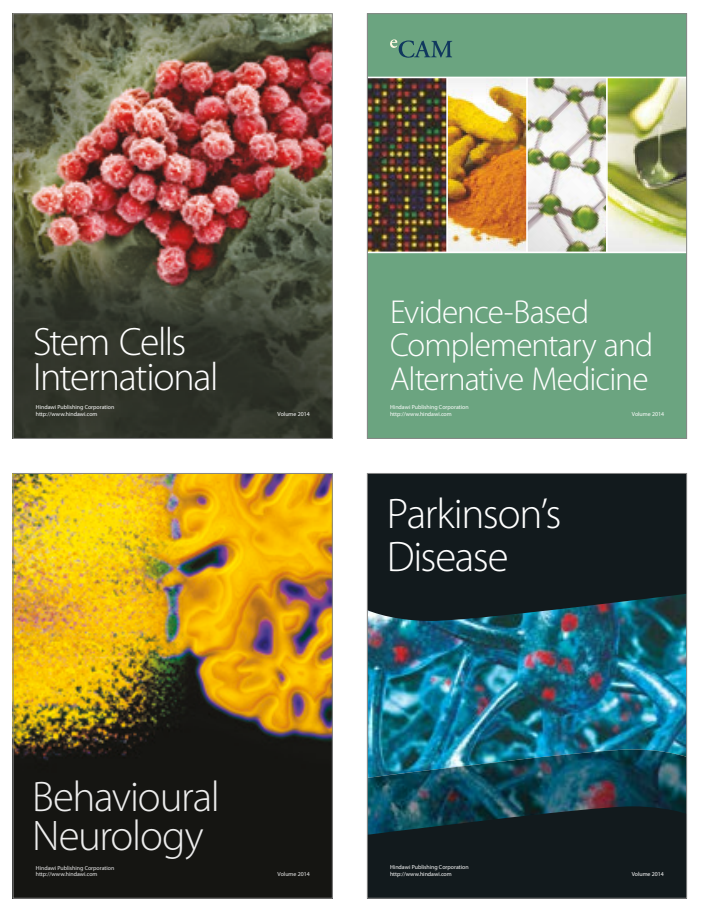
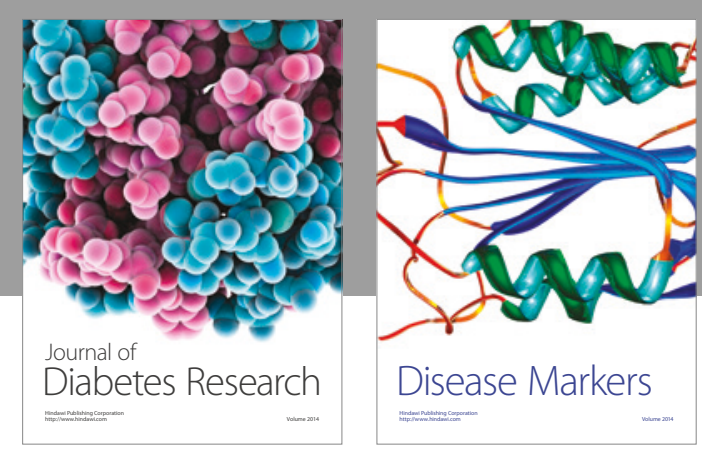

Disease Markers
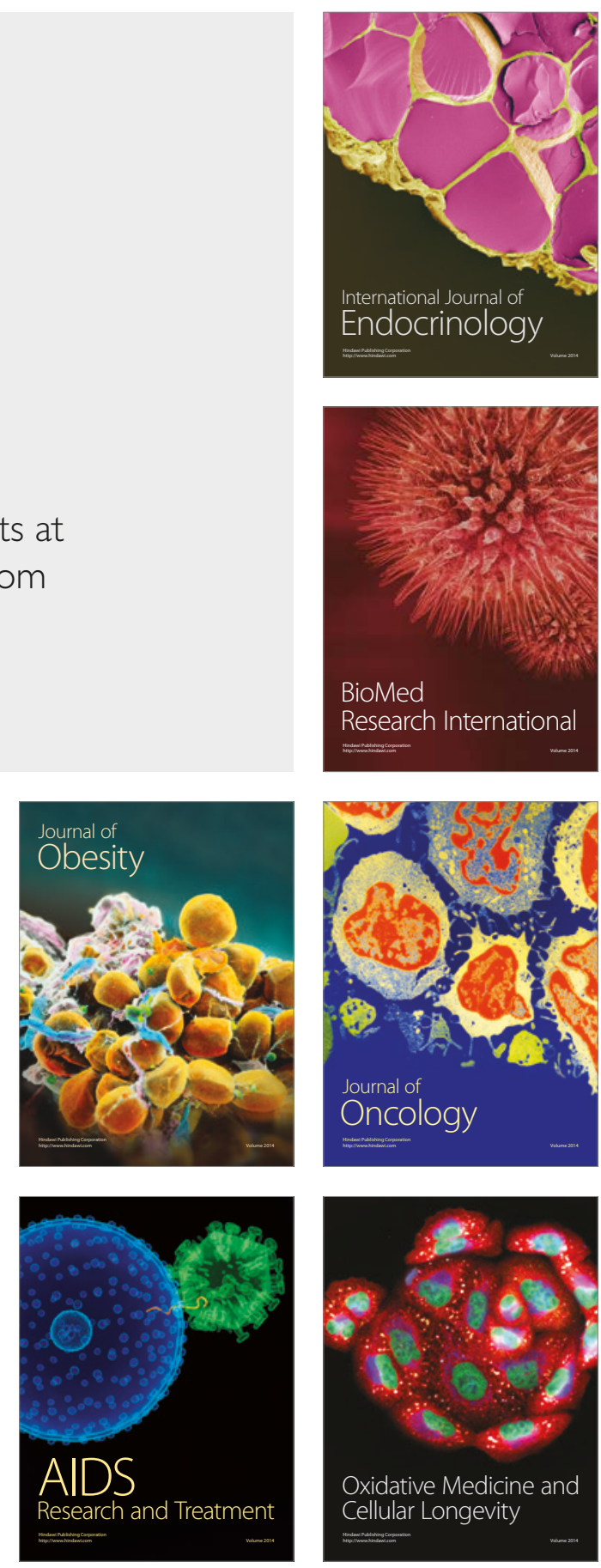